\title{
Functional Constipation among Elderly in Assiut Geriatric Clubs
}

\author{
Asmaa Gomaa Ragab ${ }^{1}$, Safaa Ahmed Kotb ${ }^{2}$, Rabaa Hamed Hassanein ${ }^{3}$, Hanaa Moukhtar Ibrahim ${ }^{4}$ \\ ${ }^{1 .}$ Nursing Master degree in Gerontological Nursing Nurse Specialist Professional nurse at Assiut, University, \\ Hospital, Egypt. \\ 2. Professor of Community Health Nursing Faculty of Nursing Assiut University, Egypt. \\ ${ }^{3 .}$ Professor of Community Health Nursing Faculty of Nursing Assiut University, Egypt. \\ ${ }^{4 .}$ Lecturer of Gerontological Nursing Faculty of Nursing Assiut University, Egypt.
}

\begin{abstract}
Constipation is a public health problem among elderly people and may lead to complications if left untreated. Aim: assess of functional constipation criteria among elderly. Research design: descriptive research design was used. Setting: This research was carried out in Assiut geriatric clubs. Sample: A study sample includes 200 elderly people. Tools: four tools have been used: $\mathbf{1}^{\text {st }}$ tool: included three parts: Part (1) socio-demographic data. Part (2) studied elderly medical history. Part (3): includes knowledge about constipation. $2^{\text {nd }}$ tool: assessment functional constipation in elderly. $3^{\text {rd }}$ tool includes two parts: Part (1) Assessment of constipation symptoms. Part (2): The standard diary $\mathbf{4}^{\text {th }}$ tool: contains two parts: Part (1): Physical Activity Scale for the Elderly. Part (2): 3-day foodrecall diary. Results: The age of studied elderly mean \pm SD was $64.67 \pm 2.64$. It was found that $72.5 \%$ of studied elderly had a functional constipation there was statistical significant between functional constipation, sitting, light activity and foods that help prevent constipation. Conclusion: Increase symptoms of functional constipation in elderly with low knowledge level of physical activity, fluids and fibers intake. Recommendations: Health education program for elderly with functional constipation about improve of physical activity and dietary intake.
\end{abstract}

\section{Keywords: Activity, Diet, Elderly, Functional Constipation \& Knowledge.}

\section{Introduction}

According to the Central Intelligence Agency (2020), Egypt's elderly population has reached 6.5 million and will also rise to 18.1 million elderly in 2050, the Egyptian life expectancy for 2019 is 71.90 years: 68.2 for men and 73.0 for women. Moreover, aging reflects the accumulation over time of changes in a human being, including physical, emotional, and social changes. It is one of the major risk factors identified for most human diseases. While old age starts, it cannot be defined uniformly because it varies according to the context. The United Nations has accepted that $65+$ years will generally be defined as old age, and this is the first attempt to define old age globally (Ouchi, et al., 2017, WHO, 2016 \& Dillin, et al., 2014). Constipation defined as difficult, incomplete and irregular bowel movements. It is the most common digestive problem which it considers a symptom than a disease (Alimoradzadeh, et al., 2017).

Constipation represents one of physiological health problem which affected elderly people than younger individuals. In addition, it is noted that the prevalence of constipation increases with age, especially for those over 65 years of age. Also chronic constipation affects $17-40 \%$ of the elderly, which interferes with their quality of life (Farahat \& et al 2019).

Constipation in older people can result from anatomical and physiological changes. Motor changes can directly influence the role of smooth muscles or via visceral innervation. Anatomical modifications can lead to internal thickening and external anal sphincter thinning. Neurological changes can decrease the electro-sensitivity of the mucosa, the sensation of rectal distention, and somatic motor nerve function (Nebhinani \& Suthar 2017).

Clinical criteria for functional constipation (FC) in elderly consists of $<3$ bowel movements a week, pressure, lumpy or rough stools, anorectal obstruction sensation, incomplete defecation and manual manoeuvring. Almost all symptoms are subjective. This is so-called self-defend constipation (Levin, 2019).

The nurse should be encouraging the elderly to increase fluid intake approximately; 2000 to 3000 $\mathrm{mL} /$ day, medically contraindicated, if not. Take at least $20 \mathrm{~g}$ of dietary fiber (e.g., raw fruits, fresh vegetables, whole grains) a day to support the patient. Urge the patient to do some exercise and physical activity. Consider isometric abdominal and gluteal exercises, encourage a quarterly deadline for removal (Wayne, 2016).

\section{Significance of the study}

In people aged 65 and over, the prevalence of constipation is between 30 percent and 40 percent, which poses an economic burden on patients and health care providers. Chronic functional 
constipation(FC) decreases the quality of life of the elderly and, with advancing age, poses many complex health issues (Cevik \& Zaybak, 2018).

Aim of the study:

The aim of the study was to assess functional constipation among elderly people at Assiut Geriatric clubs.

\section{Research questions}

1.Is there a low level of knowledge about functional constipation?

2.Do awareness and functional constipation have a good relationship?

3.Is there a positive relationship between both functional constipation and foods that help avoid constipation?

\section{Subjects \& Method}

Research design: descriptive research design was used.

Setting: This research has been performed in Assiut geriatric clubs namely (legitimacy Assembly and Islamic hub of culture).

Sample: Convenient sample (total coverage of all elderly members who agree to participate in the study) 50 legitimacy Assembly club and 150 in Islamic cultural club totally 200 elderly to assess the functional constipation.

Inclusion criteria:

- People 60 years and old

- The total number of the elderly legitimacy Assembly Club and Islamic cultural club

\section{Exclusion Criteria:}

- Previous intestinal surgery

- Use laxatives

\section{Tools of the study:}

Tool (1): self-administered questionnaire, Part (I): It includes socio demographic data as, age, sex, residence, marital status, income, occupation, and level of education and social level (El-Gilany. et al., 2012).

Part (II): This involves the medical history of the elderly, such as hypertension, diabetes, atherosclerosis and renal failure, cardiovascular disease and the medicine used.

Part (III): question to assess elderly knowledge's about constipation such as definition, symptoms, risk factors, medication, and preventing of FC as mobility, exercise, diet, and fluid intake.

Scoring system: incorrect 1 and correct 2 for the definition 2 , risk factors 16 , symptoms 12 , prevention 12 , complications 10 and management 12 the total was 64 . Less than $60 \%$ (38 poor), $60-<75 \%$ (38 48 fair) \& $75 \%$ (48) and more is good
Tool (2): To assess the functional constipation in elderly:

According to Rome II criteria: an elderly person may have constipation, if he has included two or more of the following points, straining in more than $25 \%$ defecations, lumpy or stiff stools in more than $25 \%$ of defecations, the sensation of incomplete evacuation in more than $25 \%$ defecations, anorectal obstruction, or blockage feeling in more than $25 \%$ of defecations and manual maneuvers to encourage more than 25 percent of defecations (e.g. digital evacuation, pelvic floor support) (Nour-Eldein, et al., 2014).

Tool (3):

Part I: Patient Assessment of Constipation Symptoms (PAC-SYM) Questionnaire used to measure participant symptoms as absent, mild, moderate, severe, or very severe on the 5 Likert scale, the score included 12 items comprising three abdominal subscales (four items), rectal subscales (three items) and stool subscales (five items) (Nour-Eldein, et al., 2014).

Part II: The Standard Diary

It is a regular constipation diagnosis tracking chart that tracks the number of defecations, the volume of feces, the consistency of feces, the strain during defecation, and the feeling after defecation of not having completely evacuated. According to the consistency of feces, the scale is graded from 1 to 5 : small and hard like marbles (1); bulky and hard (2); regular (3); soft (4) and watery (5). Thus, with looser fecal consistency, the score grows. Straining is scored from 1 to 4 during defecation: defecation without straining (1); At the beginning of defecation, strain (2); strain halfway through defecation (3); and strain throughout defecation (4). After defecation, the sense of not having completely evacuated was scored from 0 to 1: there was no such feeling (0), and there was such a feeling (1). The amount of feces was scored from 1 to 3: the amount of feces was smaller than the previous evacuation (1); regular (2) and large quantities of feces (3). The number of evacuations per day has also been reported (Pamuk, et al., 2003).

Tool (4):

Part I: Physical Activity Scale for the Elderly (PASE) it is a brief 10-item questionnaire that measures the past week is physical activity. The frequency of these events is graded as never [0], seldom (1-2 days/week [1]), sometimes (3-4 days/week [2]), and often (5-7 days/week [3]). The length is also graded as less than 1 hour [0], 1 to 2 hours [1], 2 to 4 hours [2], and more than 4 hours a week [3]. The final PASE activity score is calculated by multiplying by an item weight the amount of time spent on each activity (hr./week) (Colleen, 2012). 
Part II: 3-day food-recall diary.

Records have been done for everything of eating and drinking for three days (2 weekdays and 1 weekend day). Both meals, snacks, and drinks are included. Please select days that are representative of existing eating habits for the elderly (Vargas-García, \&Vargas-Salado, 2013).

\section{Validity:}

The tools were transferred to Arabic language and reviewed to ascertain their validity by five experts in the community and gerontological health nursing, who checked the method for clarification, validity, completeness, comprehension and applicability.

Reliability: was measured using cronbachs' Alpha test on $10 \%$ of cases it was 0.887 for knowledge, 0.692 for functional constipation, 0.963 for severity, and 0.668 for physical activities, 0.741 for diet.

Pilot study:

Before the start of data collection, pilot study was performed on 20 of elderly (10\%) in a selected setting to examine the applicability, and clarity of the developed tools. It was not excluded from the study.

\section{Method}

1.Administrative phase: An official letter from the Dean of the Faculty of Nursing was given to the heads of geriatric clubs in the town of Assist to permit for the researcher to carry out this study with the elderly participants. This letter contained a permit for the research to be carried out and clarified the aim of the study.

2.Ethical consideration: the Ethical Committee at the Faculty of Nursing has accepted the plan for study. There is no danger to the subject of the research during the implementation of the study. Patients were directed by their right to withdraw from the research at any time. Confidentiality and anonymity was assured. The study was followed common ethical principle in clinical research.

3.Data collection phase: An explanation of the purpose of the research was done to chiefs of geriatric clubs. Also the goal of the study was clarified to studied elderly to gain their cooperation before starting data collection. The researcher started to collect data from 11 June 2019 to 11 August 2019. Assessment was done on all the study sample (200) elderly participants. The data was collected 3 days per week at evening time, average number of elderly met beer day 7-9. Sometimes the interview questionnaire filled by the elderly themselves and others by the researcher. The length of each interview took from 30-35 minutes. The researcher was available in club to answer any questions.
4.Statistical analysis: The information obtained was checked, prepared, coded, evaluated and tabulated for computer entry. Descriptive statistic (percentages, means and standard deviations) were done using computer program SPSS version 26. The one-way variance analysis (ANOVA) test used to compare means is used. The F-test is test statistic resulting from an ordinary one-way analysis of variance (ANOVA). F-test is used to test differences in means in the three phase's pre, post, and follow-up. And Chi-square test were used for measures of significance. The $\mathrm{P}$ value is important at $\mathrm{P}<0.05$ and the $\mathrm{P}<0.01$ value is highly significant. 


\section{Results}

Table (1): Socio demographic characteristics among elderly participants in Geriatric clubs at Assiut City, 2019

\begin{tabular}{|c|c|c|}
\hline Socio demographic data & No.(200) & $\%$ \\
\hline - Age/(years): & & \\
\hline$-60-<65$ & 106 & 53.0 \\
\hline$-65-<70$ & 90 & 45.0 \\
\hline$-\geq 70$ & 4 & 2.0 \\
\hline Mean \pm SD & \multicolumn{2}{|c|}{$64.67 \pm 2.64$} \\
\hline - Sex: & & \\
\hline - Male & 14 & 7.0 \\
\hline - Female & 186 & 93.0 \\
\hline - Marital status: & & \\
\hline - Married & 122 & 61.0 \\
\hline - Widow & 75 & 37.5 \\
\hline - Divorced & 3 & 1.5 \\
\hline - Level of education: & & \\
\hline - Primary education & 16 & 8.0 \\
\hline - Preparatory education & 13 & 6.5 \\
\hline - Secondary (general \& technical of 3 or 5 years) & 132 & 66.0 \\
\hline - University graduate & 39 & 11.5 \\
\hline - Past Occupation: & & \\
\hline - Non-working/ house wife & 52 & 26.0 \\
\hline - Employee & 139 & 69.5 \\
\hline - Technical & 9 & 4.5 \\
\hline - Residence: & & \\
\hline - Rural & 6 & 3.0 \\
\hline - Urban & 194 & 97.0 \\
\hline - Living with: & & \\
\hline - Alone & 54 & 27.0 \\
\hline - With their family & 146 & 73.0 \\
\hline - Social level & & \\
\hline - Low & 45 & 22.5 \\
\hline - Middle & 96 & 48.0 \\
\hline - High & 59 & 29.5 \\
\hline
\end{tabular}

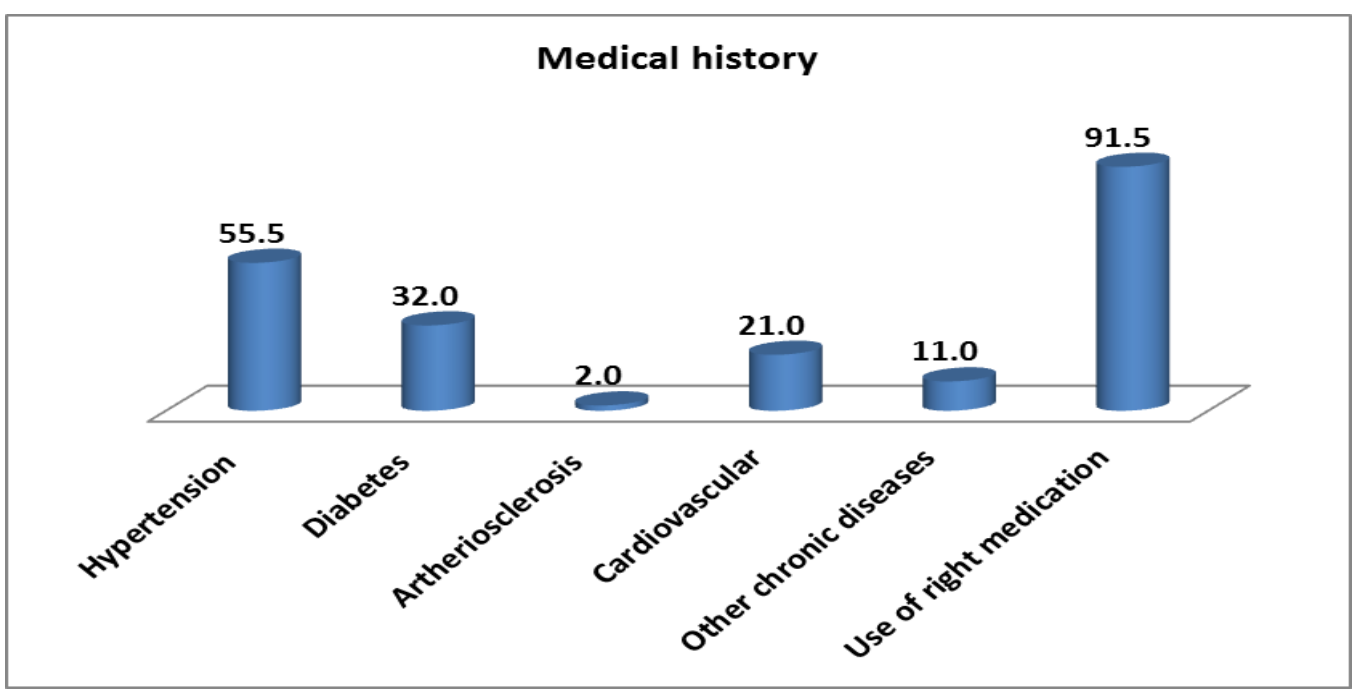

Figure (1): Distribution of the elderly participants regarding their medical history in Geriatric clubs at Assiut City, 2019 


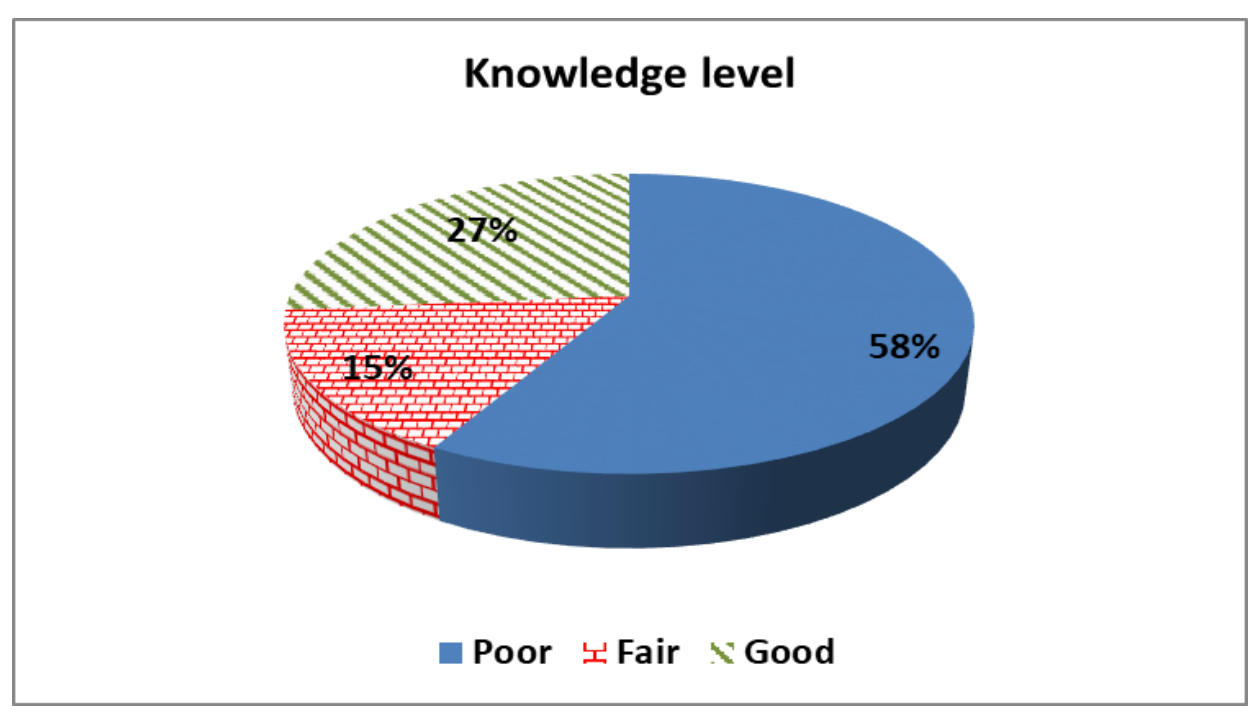

Figure (2): Distribution of the studied elderly regarding their total knowledge scores about constipation in Geriatric clubs at Assiut City, 2019

Table (2): Distribution of elderly participants regarding their symptoms of functional constipation in Geriatric clubs at Assiut City, 2019

\begin{tabular}{|l|c|c|}
\hline \multicolumn{1}{|c|}{ Items } & No. (200) & $\%$ \\
\hline In more than 25 percent of defecations, strain & 147 & $\mathbf{7 3 . 5}$ \\
\hline More than 25 percent of defecations include lumpy or hard stools & 91 & 45.5 \\
\hline Incomplete evacuation in more than 25\% of defecation sensations & 96 & $\mathbf{4 8 . 0}$ \\
\hline Anorectal obstruction or blockage feeling in over 25 percent of defecations & 95 & 47.5 \\
\hline $\begin{array}{l}\text { Manual maneuvers (e.g. digital evacuation, pelvic floor support) to promote } \\
\text { defecation by more than 25\% }\end{array}$ & 34 & $\mathbf{1 7 . 0}$ \\
\hline
\end{tabular}

\#More than one answer

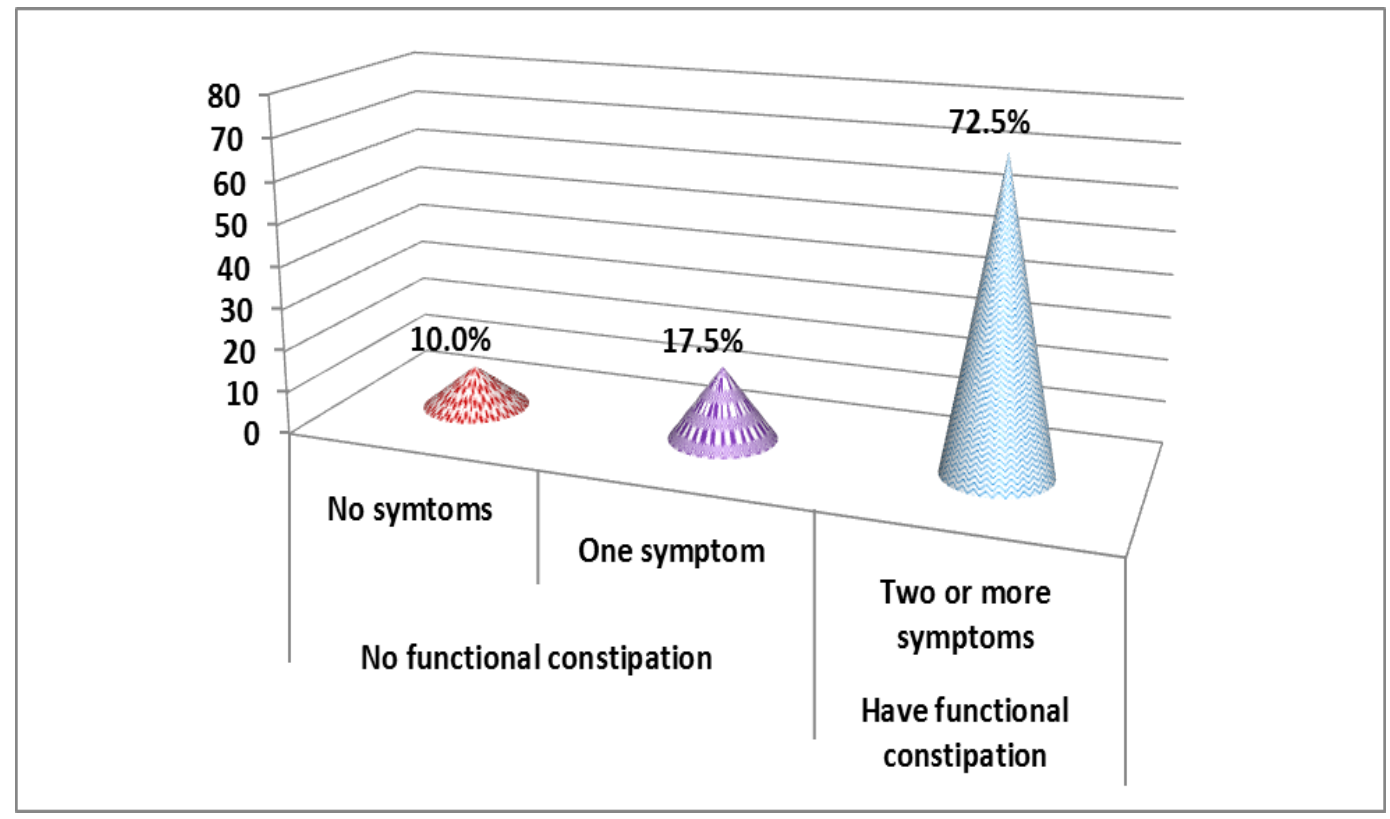

Figure (3): prevalence of functional constipation of the studied elderly in Geriatric clubs at Assiut

City, 2019 
Table (3): Distribution of elderly participants regarding their daily monitoring for symptoms of constipation (Standard diary) in Geriatric clubs at Assiut City, 2019

\begin{tabular}{|l|c|c|}
\hline Items & No.(200) & $\mathbf{\%}$ \\
\hline Consistency of feces & & \\
\hline Small and hard like marbles & 75 & 37.5 \\
\hline Bulky and hard & 64 & 32.0 \\
\hline Normal & 61 & 30.5 \\
\hline Straining during defecation & & \\
\hline Defecation with no straining & 50 & 25.0 \\
\hline At the beginning of defecation & 53 & 26.5 \\
\hline Halfway at through defecation & 30 & 15.0 \\
\hline Throughout defecation & 67 & 33.5 \\
\hline Feeling uncompleted evacuated after defecation & & \\
\hline Yes & 104 & $\mathbf{5 2 . 0}$ \\
\hline No & 96 & 48.0 \\
\hline The amount of feces & & \\
\hline Smaller than the previous evacuation & 131 & $\mathbf{6 5 . 5}$ \\
\hline Regular & 67 & 33.5 \\
\hline Large amount & 2 & 1.0 \\
\hline
\end{tabular}

Table (4): Distribution of elderly participants regarding their severity of constipation symptoms in Geriatric clubs at Assiut City, 2019

\begin{tabular}{|c|c|c|}
\hline Items & No. (200) & $\%$ \\
\hline \multicolumn{3}{|l|}{ Severe symptoms related to abdomen } \\
\hline Absent & 43 & 21.5 \\
\hline Mild & 22 & 11.0 \\
\hline Moderate & 71 & 35.5 \\
\hline Sever & 64 & 32.0 \\
\hline \multicolumn{3}{|l|}{ Severe symptoms related to rectum } \\
\hline Absent & 50 & 25.0 \\
\hline Mild & 43 & 21.5 \\
\hline Moderate & 89 & 44.5 \\
\hline Sever & 18 & 9.0 \\
\hline \multicolumn{3}{|l|}{ Severe symptoms related to stool } \\
\hline Absent & 45 & 22.5 \\
\hline Mild & 10 & 5.0 \\
\hline Moderate & 16 & 8.0 \\
\hline Sever & 129 & 64.5 \\
\hline
\end{tabular}

Table (5): Distribution of elderly participants regarding their total activities during the last 7 days in Geriatric clubs at Assiut City, 2019

\begin{tabular}{|l|c|c|}
\hline \multicolumn{1}{|c|}{ Items } & No. (200) & \% \\
\hline Sitting activities & & \\
\hline Seldom (1-2 days) & 55 & 27.5 \\
\hline Sometimes (3-4 days) & 141 & 70.5 \\
\hline Often & 4 & 2.0 \\
\hline Light activities & & \\
\hline Never (1-2 days) & 17 & 8.5 \\
\hline Seldom & 183 & $\mathbf{9 1 . 5}$ \\
\hline Moderate activities & & \\
\hline Never (1-2 days) & 4 & 2.0 \\
\hline Seldom & 196 & 98.0 \\
\hline Strenuous activities & & \\
\hline Never (1-2 days) & 188 & 94.0 \\
\hline Seldom & 12 & 6.0 \\
\hline
\end{tabular}




\begin{tabular}{|l|c|c|}
\hline \multicolumn{1}{|c|}{ Items } & No. (200) & \% \\
\hline Muscle strength activities & & \\
\hline Never (1-2 days) & 191 & 95.5 \\
\hline Seldom & 9 & 4.5 \\
\hline Household activity & & \\
\hline No & 8 & 4.0 \\
\hline Yes & 192 & $\mathbf{9 6 . 0}$ \\
\hline
\end{tabular}

Table (6): Distribution of elderly participants regarding their foods that have been eaten during the last three days, in Geriatric clubs at Assiut City 2019

\begin{tabular}{|l|c|c|}
\hline \multicolumn{1}{|c|}{ Items } & No. (200) & \% \\
\hline Foods helps to prevent constipation & & \\
\hline Water & & 60.0 \\
\hline Less than recommended & 120 & 40.0 \\
\hline Recommended amount & 80 & \\
\hline Green fruit or vegetable & 187 & $\mathbf{9 3 . 5}$ \\
\hline Less than recommended & 13 & 6.5 \\
\hline Recommended amount & & \\
\hline Fenugreek, herbs and soup & 182 & 91.0 \\
\hline Less than recommended & 18 & 9.0 \\
\hline Recommended amount & & \\
\hline Browne bread & 6 & 3.0 \\
\hline Less than recommended & 194 & $\mathbf{9 7 . 0}$ \\
\hline Recommended amount & & \\
\hline Cocked vegetable & 49 & 24.5 \\
\hline Less than recommended & 151 & 75.5 \\
\hline Recommended amount & & \\
\hline Oates, Nuts & 192 & $\mathbf{9 6 . 0}$ \\
\hline Less than recommended & 8 & 4.0 \\
\hline Recommended amount & & \\
\hline Honey & 162 & 81.0 \\
\hline Less than recommended & 38 & 19.0 \\
\hline Recommended amount & & \\
\hline Foods helps the occurrence of constipation & & \\
\hline Rice or pasta & 92 & 46.0 \\
\hline Recommended amount & 108 & $\mathbf{5 4 . 0}$ \\
\hline More than recommended amount & & \\
\hline Caffeine drinks & 39 & 19.5 \\
\hline Recommended amount & 161 & $\mathbf{8 0 . 5}$ \\
\hline More than recommended amount & 200 & $\mathbf{1 0 0 . 0}$ \\
\hline Milk product & & \\
\hline More than recommended & 67 & 66.5 \\
\hline Fried food & $\mathbf{3 3 . 5}$ \\
\hline Recommended amount & 75 & \\
\hline More than recommended amount & 125 & $\mathbf{6 2 . 5}$ \\
\hline White bread & & \\
\hline Recommended amount & & \\
\hline More than recommended amount & & \\
\hline & & \\
\hline
\end{tabular}


Table (7) Relation between functional constipation and socio demographic characteristics among elderly participants in Geriatric clubs at Assiut City 2019

\begin{tabular}{|c|c|c|c|c|c|c|c|}
\hline & \multirow{3}{*}{ Items } & \multicolumn{4}{|c|}{ Functional Constipation } & \multirow{3}{*}{$\mathbf{X}^{2}$} & \multirow{3}{*}{$\begin{array}{c}\text { P- } \\
\text { valu } \\
\text { e }\end{array}$} \\
\hline & & \multicolumn{2}{|c|}{$\begin{array}{c}\text { No constipation } \\
(\mathrm{No}=55)\end{array}$} & \multicolumn{2}{|c|}{$\begin{array}{c}\text { Constipation } \\
(\mathrm{No}=145)\end{array}$} & & \\
\hline & & No. & $\%$ & No. & $\%$ & & \\
\hline \multirow[t]{3}{*}{ Age } & $60-<65$ & 33 & 60.0 & 73 & 50.3 & \multirow{3}{*}{2.640} & \multirow{3}{*}{0.267} \\
\hline & $65-<70$ & 22 & 40.0 & 68 & 46.9 & & \\
\hline & $\geq 70$ & 0 & 0.0 & 4 & 2.8 & & \\
\hline \multirow[b]{2}{*}{ Sex } & Male & 6 & 10.9 & 8 & 5.5 & \multirow[b]{2}{*}{1.781} & \multirow{2}{*}{0.182} \\
\hline & Female & 49 & 89.1 & 137 & 94.5 & & \\
\hline \multirow{3}{*}{$\begin{array}{l}\text { Marital } \\
\text { status }\end{array}$} & Married & 39 & 70.9 & 83 & 57.2 & \multirow{3}{*}{3.388} & \multirow{3}{*}{0.184} \\
\hline & Widow & 15 & 27.3 & 60 & 41.4 & & \\
\hline & Divorced & 1 & 1.8 & 2 & 1.4 & & \\
\hline \multirow{4}{*}{$\begin{array}{l}\text { Level of } \\
\text { education }\end{array}$} & Primary education & 2 & 3.6 & 14 & 9.7 & \multirow{4}{*}{5.252} & \multirow{4}{*}{0.386} \\
\hline & Preparatory education & 3 & 5.5 & 10 & 6.9 & & \\
\hline & $\begin{array}{l}\text { Secondary (general \& } \\
\text { technical of } 3 \text { or } 5 \text { years) }\end{array}$ & 42 & 76.4 & 80 & 71.9 & & \\
\hline & University graduate & 8 & 14.5 & 31 & 21.4 & & \\
\hline \multirow{3}{*}{$\begin{array}{l}\text { Past } \\
\text { Occupation }\end{array}$} & Non-working/ house wife & 18 & 32.7 & 34 & 23.4 & \multirow{3}{*}{2.712} & \multirow{3}{*}{0.258} \\
\hline & Employee & 36 & 65.5 & 103 & 71.0 & & \\
\hline & Technical & 1 & 1.8 & 8 & 5.5 & & \\
\hline \multirow[t]{2}{*}{ Residence: } & Rural & 2 & 3.6 & 4 & 2.8 & \multirow{2}{*}{0.106} & \multirow{2}{*}{0.745} \\
\hline & Urban & 53 & 96.4 & 141 & 97.2 & & \\
\hline \multirow{2}{*}{ Living with } & Alone & 12 & 21.8 & 42 & 29.0 & \multirow{2}{*}{1.033} & \multirow{2}{*}{0.309} \\
\hline & With their family & 43 & 78.2 & 103 & 71.0 & & \\
\hline \multirow[t]{3}{*}{ Social level } & Low & 1 & 1.8 & 12 & 8.3 & \multirow{3}{*}{3.902} & \multirow{3}{*}{0.142} \\
\hline & Middle & 24 & 43.6 & 48 & 33.1 & & \\
\hline & High & 30 & 54.5 & 85 & $\begin{array}{l}58.6 \\
\end{array}$ & & \\
\hline
\end{tabular}

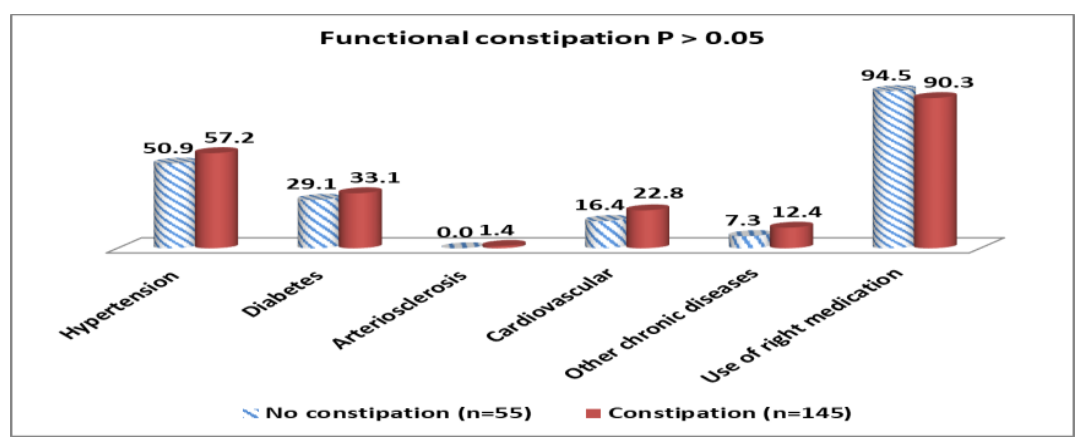

Figure (4): Relation between functional constipation and chronic disease among elderly participants in Geriatric clubs at Assiut City 2019

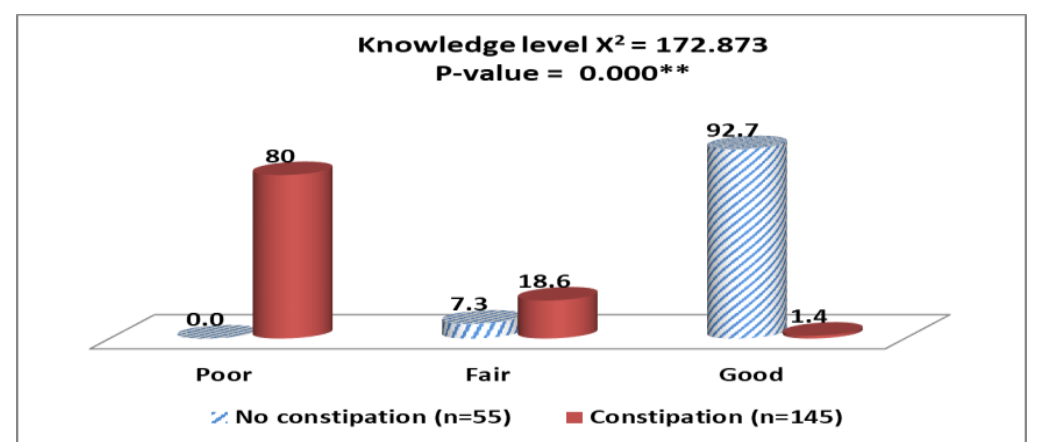

Figure (5): Relation between functional constipation and total knowledge among elderly participants in Geriatric clubs at Assiut City 2019 
Table (8) Relation between functional constipation and severity of constipation symptoms among elderly participants in Geriatric clubs at Assiut City 2019

\begin{tabular}{|c|c|c|c|c|c|c|}
\hline \multirow{3}{*}{$\begin{array}{c}\text { Severity of constipation } \\
\text { symptoms }\end{array}$} & \multicolumn{4}{|c|}{ Functional constipation } & \multirow{3}{*}{$\mathbf{X}^{2}$} & \multirow{3}{*}{ P-value } \\
\hline & \multicolumn{2}{|c|}{$\begin{array}{c}\text { No constipation } \\
(n=55)\end{array}$} & \multicolumn{2}{|c|}{$\begin{array}{c}\text { Constipation } \\
(\mathrm{n}=145)\end{array}$} & & \\
\hline & No. & $\%$ & No. & $\%$ & & \\
\hline \multicolumn{5}{|c|}{ Severe symptoms related to abdomen } & & \\
\hline Absent & 43 & 78.2 & 0 & 0.0 & \multirow[t]{4}{*}{172.642} & \multirow[t]{4}{*}{0.000} \\
\hline Mild & 12 & 21.8 & 10 & 6.9 & & \\
\hline Moderate & 0 & 0.0 & 71 & 49.0 & & \\
\hline Sever & 0 & 0.0 & 64 & 44.1 & & \\
\hline \multicolumn{5}{|c|}{ Severe symptoms related to rectum } & & \\
\hline Absent & 50 & 90.9 & 0 & 0.0 & \multirow{4}{*}{177.838} & \multirow[t]{4}{*}{$0.000^{* * 1}$} \\
\hline Mild & 5 & 9.1 & 38 & 26.2 & & \\
\hline Moderate & 0 & 0.0 & 89 & 61.4 & & \\
\hline Sever & 0 & 0.0 & 18 & 12.4 & & \\
\hline \multicolumn{5}{|c|}{ Severe symptoms related to stool } & & \\
\hline Absent & 45 & 81.8 & 0 & 0.0 & \multirow{4}{*}{200.000} & \multirow{4}{*}{$0.000^{* \pi}$} \\
\hline Mild & 10 & 18.2 & 0 & 0.0 & & \\
\hline Moderate & 0 & 0.0 & 16 & 11.0 & & \\
\hline Sever & 0 & 0.0 & 129 & 89.0 & & \\
\hline
\end{tabular}

** $P$. Value is significant at $P<0.05$

Table (9): Relation between functional constipation and total activities during the last 7 days, among elderly participants in Geriatric clubs at Assiut City, 2019

\begin{tabular}{|c|c|c|c|c|c|c|}
\hline \multirow{3}{*}{ Total activities } & \multicolumn{4}{|c|}{ Functional constipation } & \multirow{3}{*}{$\mathbf{X}^{2}$} & \multirow{3}{*}{ P-value } \\
\hline & \multicolumn{2}{|c|}{$\begin{array}{c}\text { No constipation } \\
(n=55)\end{array}$} & \multicolumn{2}{|c|}{$\begin{array}{c}\text { Constipation } \\
(n=145)\end{array}$} & & \\
\hline & No. & $\%$ & No. & $\%$ & & \\
\hline \multicolumn{7}{|l|}{ Sitting activity } \\
\hline Seldom & 11 & 20.0 & 44 & 30.3 & \multirow[t]{2}{*}{12.151} & \multirow[t]{2}{*}{0.002} \\
\hline Sometimes & 40 & 72.7 & 101 & 69.7 & & \\
\hline Often & 4 & 7.3 & 0 & 0.0 & & \\
\hline \multicolumn{7}{|l|}{ Light activity } \\
\hline Never & 1 & 1.8 & 16 & 11.0 & \multirow[t]{2}{*}{4.355} & \multirow{2}{*}{$\mathbf{0 . 0 3 7}$} \\
\hline Seldom & 54 & 98.2 & 129 & 89.0 & & \\
\hline \multicolumn{7}{|l|}{ Moderate activity } \\
\hline Never & 2 & 3.6 & 2 & 1.4 & \multirow[t]{2}{*}{1.036} & \multirow{2}{*}{0.309} \\
\hline Seldom & 53 & 96.4 & 143 & 98.6 & & \\
\hline \multicolumn{7}{|l|}{ Strenuous activities } \\
\hline Never & 49 & 89.1 & 139 & 95.9 & \multirow[t]{2}{*}{3.242} & \multirow[t]{2}{*}{0.072} \\
\hline Seldom & 6 & 10.9 & 6 & 4.1 & & \\
\hline \multicolumn{7}{|l|}{ Muscle strength activity } \\
\hline Never & 52 & 94.5 & 139 & 95.9 & \multirow[t]{2}{*}{0.161} & \multirow[t]{2}{*}{0.688} \\
\hline Seldom & 3 & 5.5 & 6 & 4.1 & & \\
\hline
\end{tabular}

** $P$. Value is significant at $P<0.05$ 
Table (10) Relation between functional constipation and foods that have been eaten during the last three days, among elderly participants in Geriatric clubs at Assiut City, 2019

\begin{tabular}{|c|c|c|c|c|c|c|}
\hline \multirow{3}{*}{ Items } & \multicolumn{4}{|c|}{ Functional constipation $(n=145)$} & \multirow{3}{*}{$\mathbf{X}^{2}$} & \multirow{3}{*}{ P-value } \\
\hline & \multicolumn{2}{|c|}{$\begin{array}{c}\begin{array}{c}\text { No constipation } \\
(n=55)\end{array} \\
\end{array}$} & \multicolumn{2}{|c|}{$\begin{array}{c}\begin{array}{c}\text { Constipation } \\
(n=145)\end{array} \\
\end{array}$} & & \\
\hline & No. & $\%$ & No. & $\%$ & & \\
\hline \multicolumn{7}{|l|}{-Foods help prevent constipation } \\
\hline \multicolumn{7}{|l|}{ Water } \\
\hline less than recommended & 25 & 45.5 & 95 & 65.5 & \multirow{2}{*}{6.688} & \multirow{2}{*}{$0.010^{*}$} \\
\hline recommended amount & 30 & 54.5 & 50 & 34.5 & & \\
\hline \multicolumn{7}{|l|}{ Green fruit or vegetable } \\
\hline less than recommended & 42 & 76.4 & 145 & 100.0 & \multirow[t]{2}{*}{36.655} & \multirow[t]{2}{*}{$0.000^{* *}$} \\
\hline recommended amount & 13 & 23.6 & 0 & 0.0 & & \\
\hline \multicolumn{7}{|l|}{ Fenugreek, herbs and soup } \\
\hline less than recommended & 37 & 67.3 & 145 & 100.0 & \multirow{2}{*}{52.148} & \multirow{2}{*}{$0.000^{* *}$} \\
\hline recommended amount & 18 & 32.7 & 0 & 0.0 & & \\
\hline \multicolumn{7}{|l|}{ Browne bread } \\
\hline less than recommended & 0 & 0.0 & 6 & 4.1 & \multirow[t]{2}{*}{2.346} & \multirow[t]{2}{*}{0.126} \\
\hline recommended amount & 55 & 100.0 & 139 & 95.9 & & \\
\hline \multicolumn{7}{|l|}{ Cocked vegetable } \\
\hline less than recommended & 13 & 23.6 & 36 & 24.8 & \multirow[t]{2}{*}{0.031} & \multirow[t]{2}{*}{0.861} \\
\hline recommended amount & 42 & 76.4 & 109 & 75.2 & & \\
\hline \multicolumn{7}{|l|}{ Oates, Nuts } \\
\hline less than recommended & 47 & 85.5 & 145 & 100.0 & \multirow[t]{2}{*}{21.970} & \multirow[t]{2}{*}{$0.000^{* *}$} \\
\hline recommended amount & 8 & 14.5 & 0 & 0.0 & & \\
\hline \multicolumn{7}{|l|}{ Honey } \\
\hline less than recommended & 41 & 74.5 & 121 & 83.4 & 2.054 & 0.152 \\
\hline recommended amount & 14 & 25.5 & 24 & 16.6 & & \\
\hline -Foods helps the occurrence of c & & & & & & \\
\hline Rice & & & & & & \\
\hline Recommended amount & 30 & 54.5 & 62 & 42.8 & 2330 & 0135 \\
\hline More than recommended amount & 25 & 45.5 & 83 & 57.2 & 2.250 & 0.155 \\
\hline Caffeine drinks & & & & & & \\
\hline Recommended amount & 21 & 38.2 & 18 & 12.4 & 16.867 & $0.000^{* * *}$ \\
\hline More than recommended amount & 34 & 61.8 & 127 & 87.6 & & \\
\hline Milk product & & & & & & \\
\hline More than recommended amount & 55 & 100.0 & 145 & 100.0 & - & - \\
\hline Fried food & & & & & & \\
\hline Recommended amount & 40 & 72.7 & 93 & 64.1 & 1.321 & 0.250 \\
\hline More than recommended amount & 15 & 27.3 & 52 & 35.9 & & \\
\hline White bread & & & & & & \\
\hline Recommended amount & 31 & 56.4 & 44 & 30.3 & 11.518 & $0.001^{*}$ \\
\hline More than recommended amount & 24 & 43.6 & 101 & 69.7 & & \\
\hline
\end{tabular}

** P. Value is significant at $P<0.05$

Table (1): Shows the sociodemographic characteristics of the elderly studied. It was noted that the age group between $60-<65$ years represent more than half $(53.0 \%)$ with mean \pm S.D. was $64.67 \pm 2.64$. Also $93.0 \%$ and $97.0 \%$ of them were female and from urban, also and $61.0 \%, 66.0$ and $69.5 \%$ of them were married, secondary education and employee. in addition $73.0 \%$ and $48.0 \%$ of them live with their family and hade middle social level.

Figure (1): This figure showed that $55.5 \%$ of studied elderly had history of hypertension while $2.0 \%$ had atherosclerosis.

Figure (2): Also that $58.0 \%$ of studded elderly had poor knowledge score while $27.0 \%$ of them had good knowledge scores about constipation 
Table (2): This table showed that $73.5 \%$ of studied elderly had straining in more than $25 \%$ of defecations and $48.0 \%$ of them were in more than $25 \%$ of defecations, the feeling of incomplete evacuation. While $17.0 \%$ of them reported manual maneuvers to promote more than 25 percent of defecations (e.g., digital evacuation, pelvic floor support).

Figure (3): Illustrate that $72.5 \%$ of studied elderly had functional constipation with two or more symptoms.

Table (3): Reported $65.5 \%$ of studied elderly the amount of feces was less than that of the previous evacuation. While $52.0 \%$ of them reported that had feeling uncompleted evacuated after defecation.

Table (4): Also $64.5 \%$ and $32.0 \%$ respectively of studied elderly had severe symptoms related to stool and abdomen

Table (5): This table found that $91.5 \%$ and $98.0 \%$ of studied elderly had seldom light activities and moderate activities, while $94.0 \%$ don't do strenuous activities.

Table (6): This table reported that $93.5 \%, 91.0 \%$, 96.0 and 81.0 respectively of the studied elderly reported take green fruit or vegetable, fenugreek, herbs and soup, oates, nuts and honey less than recommended. While $100.0 \%$ of studied sample take milk product more than recommended and $54.0 \%$, $80.5 \%, 33.5 \%$ and 62.5 of them take rice or pasta, caffeine drinks, fried food and white bread more than recommended amount.

Table (7): This table illustrated that $97.0 \%$ and 94.5 $\%$ of studied sample hade constipation were female and were from urban. This also no statistical significant difference at all times.

Figure (4): Show that the no statistical significant between functional constipation and chronic disease among elderly participants.

Figure (5): While $80.0 \%$ of studied elderly with constipation hade poor knowledge score with highly statistical significant difference at $\mathrm{P}=0.000$

Table (8): This table showed that $89.0 \%, 44.1 \%$ and 12.4 of studied elderly with constipation had severe symptoms related to stool, abdomen, and rectum with statistical significant difference at $\mathrm{P}=0.000$ at all items.

Table (9): Presented that there was a statistically significant difference between functional constipation and activities during the last 7 days with $\mathrm{P}=0.002$, 0.037 at items named sitting activity and light activity.

Table (10): Presented that there were statistically significant differences between functional constipation and foods eating during the last three days with $\mathrm{P}=0.000$ at items name green fruit or vegetable, fenugreek, herbs and soup, oates, Nuts and $\mathrm{P}=0.010$ at drinking water less than recommended.
While $\mathrm{P}=0.000,0.001$ take caffeine drinks and white bread more than recommended amount

\section{Discussion:}

Constipation is a prolonged, multifunctional condition that affects about $20 \%$ of the world's total population and is more common in females and the elderly. Its prevalence ranges from $7.72 \%$ to $42.86 \%$ in people older than 70 years (Gomes et al 2019). The aim of the study was to assess functional constipation among elderly people at Assiut Geriatric clubs. With regard to socio-demographic characteristics, this analysis showed that more than half of the studied were elderly aged between 60-<65 year, and majority of elderly were female. Also more than half of studied samples were married, got secondary education and approximately less than three-quarters of them were employee. In addition, the vast majority of the studied samples were living in urban areas.

The current findings agree with Gomes et al., (2019) it is more commonly found in women who fined the higher prevalence in the age group of 60-69 years old, and its related factors. Moreover, Qaseem et al., (2017) found that constipation rates perform to increase gradually after the age of 50 years. Mihara et al., (2020) showed that female sex as an independent risk factor of constipation.

This result noted that vast majority of elderly participants were live in urban areas. This may be related to the natural of urban society living which characterized by limited activity level than rural society. This is similar to Yilmaz \& Asiret, (2017) who found that elderly people in the urban areas had a higher prevalence in constipation than rural areas, but there is no specific description in either domestic or international research to clarify this difference. Farahat et al (2019) who study the risk factors for constipation among elderly people attending the Damietta District family health center, and reported that elderly population in the urban areas had a higher prevalence in constipation than rural areas but there is no specific description in either domestic or international research to explain this difference. Yang et al., (2016) who showed that there was no difference in the constipation incidence between urban and rural areas.

Traditionally, care for older persons was a duty of the Egyptian family. Family support is especially important for older people, particularly when they need assistance because of chronic illnesses and diseases that are debilitating Shaheen et al (2017). Also the present study revealed that the majority of the researchers studied sample living with their family, this may be indicated to the effect on dependent on their family to had decrease physical ability. This results disagree with, Aminu, (2019) 
who study Association between the effects of oral health and older adults' living arrangements, in their study of the association between living alone and health care utilization in older adults, mentioned that a great number of the studied elderly persons live alone. Also, this results agreed with Yan, \& Fang, (2017) who studied elder abuse and neglect in Asia, and found that the majority of elder live with their family.

The present research has shown that more than half of studied sample had lowly knowledge. Turk et al (2014) who studied General knowledge about diabetes in the elderly diabetic population in Slovenia, found that the elderly diabetic low level of general knowledge about the disease.

A symptom and not a disease is constipation. It happens if the colon absorbs too much water or if the muscle contractions of the colon are sluggish or slow, allowing the stool to pass too slowly through the colon. (Chen et al., (2017). This study presented that less than three quarter of the studied elderly had constipation with more than one symptom approximately more than two thirds of them had a straining in more than $25 \%$ of defecations.

O'Neill et al., (2017) who study Burden and risk factors for gastrointestinal symptom, found that the prevalence of functional constipation has detrimental effects on the quality of life and sleepiness during the day. Although the demographics and disease burden are identical between functional constipation and irritable bowel syndrome, although the clinical symptoms vary considerably.

Regarding the severity of constipation symptoms, the current findings show that more than one quarter of the studied elderly had severe symptoms related to abdomen and less than two quarters of the studied elderly had severe symptoms related to stool. This agreed with Dumic et al (2019) who study Older-age Gastrointestinal Tract Disorders, found the elderly constipation symptoms straining rather than decreased bowel movement frequency and abdominal pain and discomfort among elderly people.

The present study illustrated that functional constipation and activities during the last 7 days, the majority of the studied sample show in light and household activity. These results are in agreement with Gao et al., (2019) who study Exercise therapy in patients with constipation: a systematic study of randomized controlled trials and a meta-analysis. The findings of this systematic examination and metaanalysis showed that, as a way of improving the symptoms of constipation patients, exercise had important benefits.

In addition, this is similar to results reported by Forootan et al., (2018) who study chronic constipation and found that the dietary changes, increased intestinal transit times, lack of physical activity, are potential causes for the development of functional constipation in the elderly.

This study showed the statistical significant differences between functional constipation and foods that help prevent constipation during the last three days observed on green fruit or vegetable, fenugreek, herbs and soup, oates, Nuts and water less than recommended. Also between functional constipation and foods that have been eaten during the last three days take caffeine drinks and white bread more than recommended amount.

This is agreement with Emmanuel et al., (2016) who study Constipation in Older People, A Consensus Statement, It found that fiber intake was significant, particularly for the elderly, to the point that all national dietary recommendations and food guide pyramids emphasize the need to increase dietary fiber intake, such as fruits and vegetables, in order to avoid functional constipation.

Moreover, this finding supported by Haller et al., (2020) who study Nutrition Therapy for Intestinal Disorders and fiber supplementation was found to be in the form of laxative discontinuation in more than half of the fiber population.

Also in the same line with Ahn et al., (2018) Who discovered that statistical significance was provided relation between constipation and malnutrition, where the percentage of constipation was significantly lower among elderly patients who ate fruits and vegetables and those who drank more than five cups of fluids per day. This may be attributed to low intake of fluid, which is associated with slow colonic transit and low stool production.

This is in agreement with Martínez-Martínez et al., (2017) and all older people individuals evaluated as having a mean intake of fiber below the recommended rate were considered constipated as compared with others. Moreover, Lopes et al., (2019) found that higher fiber intake was correlated with a decreased risk of constipation after multiple factors were regulated.

\section{Conclusion}

Based on the results findings, having concluded that, Increase symptoms of functional constipation in elderly with low level of knowledge about physical activity and suitable dietary intake of fluids and fibers

\section{Recommendations:}

- Health education program about functional constipation, dietary intake and physical activities

- Provide a simplified booklet about functional constipation (symptoms, physical activity and suitable diet) in the library of geriatric clubs 
- Further research about the effect of nursing intervention fiber intake was decreased risk of constipation.

\section{Reference}

- Ahn J., Park J, \& Kim C. (2018): Effects of an individualised nutritional education and support programme on dietary habits, nutritional knowledge and nutritional status of older adults living alone. Journal of clinical nursing, 27(9-10), 2142-2151

- Alimoradzadeh R, Mokhtare M, \& Agah Sh, (2017): [Comparing the Prevalence of Constipation Risk Factors in the Elderly With and Without Constipation in Hazrat-e Rasoul (PBUH) Hospital (Persian)]. Scale for health research in Egypt. Iranian Journal of Ageing.; Volume 12, No (1): Page 78-89.

- Aminu A. (2019): Adult Dental Health Survey 2009: association between oral health outcomes and living arrangements of older adults in the UK. British dental journal, 227(2): 115-120.

- Central Intelligence Agency, (2020): Egypt Demographics Profile 2020,Availableat:https://www.indexmundi.com/eg ypt/demographics_profile.html.

- Cevik K, \& Zaybak A, (2018): The Effect of Reflexology on Constipation in the Elderly, International Journal of Caring Sciences JanuaryApril Volume $11 \mid$ Issue 1|Page 309

- Chen J., Yu Y, Yang Z, Yu W., Chen W., Yu H, \& Chen J, (2017): Intraluminal pressure patterns in the human colon assessed by highresolution manometry. Scientific reports, 7, 41436

- Colleen C, (2012):"Methods of physical activity assessment in older adults" Graduate Theses and Dissertations. 12809. Available at https://lib.dr.iastate.edu/etd/12809.

- Dillin,A., Gottschling, DE., \& Nyström, T., (2014): "The good and the bad of being connected: the integrons of aging". Curr Opin Cell Biol. 26: 107-12.

- Dumic I, Nordin T, Jecmenica M, Lalosevic M, Milosavljevic T, \& Milovanovic T, (2019): Gastrointestinal Tract Disorders in Older Age : Canadian Journal of Gastroenterology and Hepatology, $19 \quad$ pages https://doi.org/10.1155/2019/6757524

- El-Gilany A, El-Wehady \& El-Wasify, M (2012): Updating and validation of the socioeconomic status EMHJ: Volume 18, No. (9) Page 962-8.

- Emmanuel A , Raso F, Neri M , Petersen K , Rey E, and Rogers J, (2016): Constipation in older people: A consensus statement December
International Journal of Clinical Practice 71(1) DOI: $10.1111 /$ ijcp.12920

- Farahat T, El-Esrigy F, \& Salama W, (2019): Risk factors for constipation among elderly attending family health center in Damietta District, Damietta Governorate, Egypt | Volume : 32| Issue : 1 | Page : 145-150

- Forootan M, Bagheri N, \& Darvishi M, (2018): Chronic constipation: A review of literature. Medicine, 97(20.

- Gao R, Tao Y, Zhou C, Li J, Wang X, Chen L, \& Guo L, (2019): Exercise therapy in patients with constipation: a systematic review and metaanalysis of randomized controlled trials. Scandinavian journal of gastroenterology, 54(2), 169-177.

- Gomes S, Duarte Y, \& Santos J, (2019): Intestinal constipation in the elderly and associated factors - SABE Study J. Coloproctol. (Rio J.) vol.39 no.2

- Haller E, Issokson K, Lebovits J, \& Scarlata K, (2020): Nutrition Therapy for Intestinal Disorders. Geriatric Gastroenterology, 1-23

- Levin MD, (2019): Pathophysiology of the functional constipation in elderly State Geriatric Center, Netanya, ; 38: 53-57. Available at http://www.pelviperineology.org.http://dx.doi.org/ 10.34057/PPj.2019.38.02.006

- Lopes S., Miszputen S., Sachs A, Lima M., \& AMBROGINI JR, (2019): Evaluation of carbohydrate and fiber consumption in patients with irritable bowel syndrome in outpatient treatment. Arquivos de gastroenterologia, 56(1), 3-9.

- Martínez-Martínez M., Calabuig-Tolsa R, \& Cauli O. (2017): The effect of probiotics as a treatment for constipation in elderly people: a systematic review. Archives of Gerontology and Geriatrics, 71, 142-149.

- Mihara H, Murayama A, Nanjo S, Ando T, Tajiri K, Fujinami H, \& Yasuda I. (2020): Factors correlated with drug use for constipation: perspectives from the 2016 open Japanese National Database. BMC gastroenterology, 20(1), 1-8.

- Nebhinani N, \& Suthar N, ( 2017): Constipation in elderly patients with psychiatric disorders REVIEW ARTICLE, Volume : 4 | Issue : 1 | Page : 11-17.

- Nour-Eldein H, Hind M, Abdulmajeed A, \& Khaled S, (2014): The effect of lifestyle modification on severity of constipation and quality of life of elders in nursing homes at Ismailia city, Egypt. Journal of Family and Community Medicine, Vol 21 | Issue 2 | 100-106. 
- O’Neill T., Raboud J., Tinmouth J, Rourke S. B, Gardner S, Cooper C, \& OHTN Cohort Study Team. (2017): Burden and risk factors for gastrointestinal symptom distress in HIV patients in the modern antiretroviral era. AIDS care, 29(2), 156-167.

- Ouchi Y, Rakugi H, Arai H, Akishita M, Ito H, \& Toba $K$,(2017): REPORT OF THE COMMITTEE Redefining the elderly as aged 75 years and older: Proposal from the Joint Committee of Japan Gerontological Society and the Japan Geriatrics Society17: 1045-1047

- Pamuk ON, Pamuk GE, \& Celik AF, (2003): Revalidation of description of constipation in terms of recall bias and visual scale analog questionnaire. Journal of Gastroenterology and Hepatology, 18: 1417-1422.

- Qaseem A, Wilt T., Rich R, Humphrey L., Frost J, \& Forciea M. (2017): Pharmacologic treatment of hypertension in adults aged 60 years or older to higher versus lower blood pressure targets: a clinical practice guideline from the American College of Physicians and the American Academy of Family Physicians. Annals of internal medicine, 166(6), 430-437.

- Shaheen H, Badr S, \& Saleh E, (2017): Comparative study between elderly with medical problems living in endwelling houses and with families in Banha City ORIGINAL ARTICLE: | Volume : 30 | Issue : 1 | Page : 44-50

- Turk E, Palfy M, Rupe V, \& Isola A, (2014): General knowledge about diabetes in the elderly diabetic population in Slovenia Izvirni članek/Original article: available at file://C:/Users/Marwaan/Downloads/diabetesgene ralknowledge.pdf

- Vargas-García EJ, \& Vargas-Salado E, (2013): Food intake, nutritional status and physical activity between elderly subjects with and without chronic constipation. A comparative study Volume 81, No. (3): Page 199-204. 3-Day Food Intake Record. Available at file:///C:/Users/wifi/Downloads/Documents/3 Da y_Food_Intake_Record_STARFHT.pdf

- Wayne G, (2016): Constipation Nursing Care Plan. Available at https://nurseslabs.com/constipation/

- WHO (2016): Definition of an older or elderly person". Available https://en.wikipedia.org/wiki/Old_age.

- Yang X., Zhang M, Zhu H., Tang Z, Zhao D. D, Li B., \& Gabriel A, (2016): Epidemiological study: Correlation between diet habits and constipation among elderly in Beijing region. World journal of gastroenterology, 22(39), 8806.
- Yilmaz C., \& Asiret G., (2017): Identifying the Constipation Levels of Older People and their Interventions for Recovery. International Journal of Caring Sciences, 10(3), 1605-1614. 